\title{
Asistencia en Prostodoncia Removible frente a la pandemia por COVID-19
}

Profa. Dra. Mariana Seoane Campomar

Cátedra de Rehabilitación en Prostodoncia Removible II

Facultad de Odontología Udelar

ORCID: 0000-0002-8561-2553

\section{Introducción}

Desde el inicio de la infección por el virus SARS Cov-2 consensuado en diciembre de 2019, se han incrementado exponencialmente las publicaciones científicas sobre su origen, transmisibilidad, riesgo y sobrevida. Es así que, centros de referencia sanitaria en diferentes países han generado protocolos destinados a poblaciones de riesgo de transmisión como la médica y la odontológica ${ }^{(1-3)}$.

Si bien hasta el momento se sostiene que los sujetos con síntomas son los principales responsables de la transmisión comunitaria, las personas en período de incubación y/o luego de recuperarse pueden contagiar (4-7). Es por ello que, cualquier sujeto que se encuentre en la consulta es un potencial agente de transmisión (incluido el personal del equipo de salud) ${ }^{(4,8)}$.

En el actual escenario de pandemia, los protocolos recomiendan postergar los procedimientos odontológicos programados y no comprendidos dentro de la urgencia ${ }^{(8-11)}$. Publicaciones provenientes de países que atravesaron gran parte de la emergencia sanitaria brindan pautas para la realización de procedimientos de urgencia que involucran dolor y traumatismos ${ }^{(3,10)}$, pero, no profundizan en recomendaciones para procedimientos de rutina ni para urgencias que no se enmarcan en las situaciones mencionadas.

Probablemente, los tratamientos asociados al área de la Prostodoncia puedan, en su mayoría, posponerse. Sin embargo, una pandemia de meses de duración torna plausible y altamente probable que surjan situaciones de urgencia prostodóncica vinculadas fundamentalmente a la necesidad de reparar aparatos protésicos ${ }^{(12)}$. Asimismo, pueden traducirse en urgencias odontológicas ciertos sucesos inesperados en pacientes que se encuentren bajo tratamiento momentáneamente suspendido por la pandemia o en quienes requieran la realización de un nuevo aparato. En estos últimos casos se impone un diálogo sensato con el paciente, identificando riesgos, valorando beneficios y priorizando 
el rol ético y profesional del Odontólogo, así como la comunicación e información basada en la evidencia presente hasta el momento.

\section{Transmisión del SARS Cov-2 en el ámbito de la Prostodoncia}

El Odontólogo que durante la pandemia deba realizar procedimientos asociados a la Prostodoncia requiere conocer las vías de transmisión del SARS-Cov-2 (directas o indirectas), el tiempo de permanencia del virus sobre superficies inanimadas y el tiempo necesario para que un desinfectante lo inactive (13-16). Asimismo, es posible considerar que, en general, los procedimientos destinados a reparar prótesis removibles involucran el uso de instrumental semicrítico o no crítico por lo que muchos de ellos pueden desinfectarse, aunque es preferible su esterilización (17).

Si bien se asume que el mayor potencial de transmisión se concentra en la producción de aerosoles, la saliva también es un vector (18-21). El receptor para SARS Cov-2 se expresa altamente en las células epiteliales de la mucosa oral y de las de las glándulas salivales ${ }^{(20,21)}$. Además, se constata la presencia del virus en superficies inanimadas producto de su diseminación desde las vías anteriormente mencionadas ${ }^{(4)}$. Debido al uso de fómites que podrían originar rutas de diseminación, estos son aspectos que imponen asumir el principio de "precaución universal" y considerar con cautela a la urgencia prostodóncica y a la práctica general ${ }^{(15,22)}$.

En el marco de la actual pandemia, considerando que pueden presentarse urgencias que involucren reparaciones de prótesis removibles y que éstas, en su mayoría, incluyen la necesidad de realizar tanto procedimientos clínicos como paraclínicos, la siguiente revisión tiene por objetivo proponer un protocolo de actuación que focalice en la asistencia prostodóncica de urgencia pero que, a su vez, pueda aplicarse a la práctica prostodoncia removible en general.

Los demás procedimientos que también efectúa un Prostodoncista y que involucran otras áreas (Operatoria, Cirugía, entre otras) han sido descriptos en los artículos previos de esta publicación y no serán abordados aquí.

\section{Metodología}

Se realizó una búsqueda electrónica no sistematizada en las bases de datos Pubmed y Scopus a las que se sumaron publicaciones de organismos internacionales y artículos procedentes de referencias específicas. La única limitación incorporada fue "publicado en el último año". Se procuraron artículos relacionados en el área odontológica a través de los siguientes términos, 
"coronavirus" "covid", "dentistry", "dental", "prosthodontics" y "dentures". Los criterios de selección fueron: idioma inglés o español, adultos, área odontológica y prostodóncica. Fueron excluidos estudios que focalizaron en otras especialidades o se encontraron fuera del alcance de esta búsqueda.

Finalmente, el protocolo en su versión preliminar se puso a consideración de referentes académicos de Prostodoncia y sus aportes fueron incorporados.

Resultados

La búsqueda inicial arrojó 76 estudios en Pubmed y 11 en Scopus. Luego de identificar coincidencias y realizar la lectura de título y resúmenes se excluyeron 41 estudios, seleccionando para una lectura completa 38. A partir de ella, se excluyeron 23 artículos permaneciendo incluidos 14 por asociarse a la Odontología, proponer protocolos adaptables al área de la Prostodoncia y/o, involucrar a ésta en los mismos (Figura 1). En en su mayoría constituyen estudios secundarios, cartas al editor y relatos de experiencias (Tabla 1).

Figura 1: Flujograma

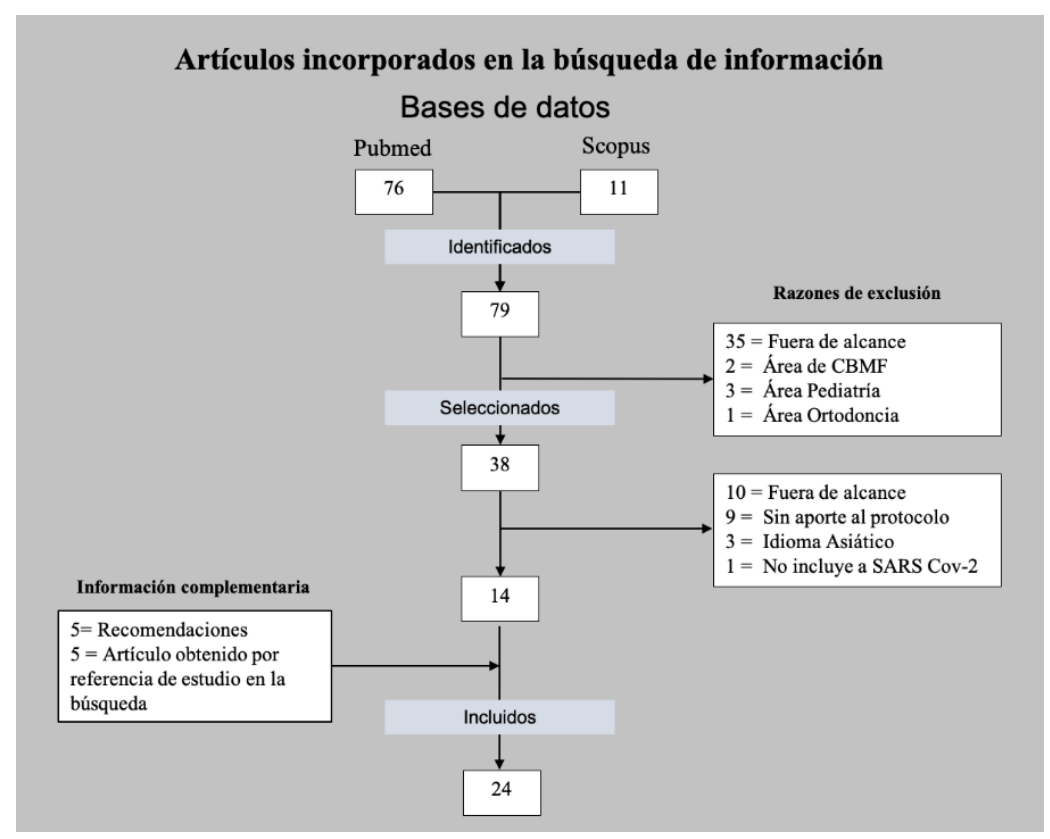


Tabla 1 - Estudios incluídos de las bases Pubmed y Scopus

\begin{tabular}{|c|c|c|c|c|}
\hline Autores & Título & Periódico & Tipo de publicación & Ciudad, País \\
\hline Bristol, M & Toothbrushing against coronavirus. & Oral Oncol & Letter to the Editor & Bristol, UK \\
\hline Alharbi A, Alharbi S, Alqaidi S. J. & $\begin{array}{l}\text { Guidelines for dental care provision during } \\
\text { the COVID-19 pandemic. }\end{array}$ & Saudi Dent & Original Article & Riyadh, Saudi Arabia. \\
\hline Aquino Canchari CR. & $\begin{array}{l}\text { Coronavirus COVID-19 y su repercusión en } \\
\text { la Odontología }\end{array}$ & Rev Cubana Estomatol & Letter to the Editor & Huancayo, Perú. \\
\hline $\begin{array}{l}\text { Ather A, Patel B, Ruparel NB, } \\
\text { Diogenes A, Hargreaves KM. }\end{array}$ & $\begin{array}{l}\text { Coronavirus Disease } 19 \text { (COVID-19): } \\
\text { Implications for Clinical Dental Care. }\end{array}$ & J Endod. & Review Article & Texas. EEUU \\
\hline Ge, Z., Yang, L., Xia, J. et al. P J. & $\begin{array}{l}\text { Possible aerosol transmission of COVID-19 } \\
\text { and special precautions in dentistry. }\end{array}$ & Zhejiang Univ. Sci. B & Review Article & $\begin{array}{l}\text { Zhejiang Province, } \\
\text { China }\end{array}$ \\
\hline Guo J, Wu H, Xie H. & $\begin{array}{l}\text { How to Deal with Suspended Oral Treatment } \\
\text { during the COVID-19 Epidemic }\end{array}$ & J Dent Res. & Short Communication & Peking, China \\
\hline $\begin{array}{l}\text { Machado RA, de Souza NL, } \\
\text { Oliveira RM, Martelli Júnior } \mathrm{H} \text {, } \\
\text { Bonan PRF. }\end{array}$ & $\begin{array}{l}\text { Social media and telemedicine for oral } \\
\text { diagnosis and counselling in the COVID- } 19 \\
\text { era. }\end{array}$ & Oral Oncol. & Letter to the Editor & San Pablo, Brasil \\
\hline Meng L, Hua F, Bian Z. & $\begin{array}{l}\text { Response to the Letter to the Editor: How to } \\
\text { Deal with Suspended Oral Treatment during } \\
\text { the COVID-19 Epidemic. PMID:32282256. }\end{array}$ & J Dent Res. & Original Article & Wuhan, China \\
\hline Peng, X., Xu, X., Li, Y. et al & $\begin{array}{l}\text { Transmission routes of } 2019-\mathrm{nCoV} \text { and } \\
\text { controls in dental practice. }\end{array}$ & Int J Oral Sci & Review Article & Chengdu, China \\
\hline $\begin{array}{l}\text { Prati C, Pelliccioni GA, Sambri V, } \\
\text { Chersoni S, Gandolfi MG. }\end{array}$ & $\begin{array}{l}\text { COVID-19: its impact on dental schools in } \\
\text { Italy, clinical problems in endodontic therapy } \\
\text { and qeneral considerations. }\end{array}$ & Int Endod J. & Letter to the Editor & Bologna, Rumania \\
\hline $\begin{array}{l}\text { Sabino-Silva, R., Jardim, A., \& } \\
\text { Siqueira, W. L. }\end{array}$ & $\begin{array}{l}\text { Coronavirus COVID-19 impacts to dentistry } \\
\text { and potential salivary diagnosis. }\end{array}$ & Clinical oral investigations & Letter to the Editor & Saskatchewan, Canada \\
\hline $\begin{array}{l}\text { Spagnuolo, G., De Vito, D., Rengo, } \\
\text { S., \& Tatullo, M. }\end{array}$ & $\begin{array}{l}\text { COVID-19 Outbreak: An Overview on } \\
\text { Dentistry }\end{array}$ & $\begin{array}{l}\text { International Journal of } \\
\text { Environmental Research } \\
\text { and Public Health, }\end{array}$ & Review Article & Naples, Italy \\
\hline Yang Y, Zhou Y, Liu X, Tan J. & $\begin{array}{l}\text { Health services provision of } 48 \text { public tertiary } \\
\text { dental hospitals during the COVID-19 } \\
\text { epidemic in China. }\end{array}$ & Clin Oral Investig. & Short Communication & Beijing, China \\
\hline van Doremalen, Neeltje et al & $\begin{array}{l}\text { Aerosol and Surface Stability of SARS-CoV- } \\
2 \text { as Compared with SARS-CoV-1 }\end{array}$ & $\begin{array}{l}\text { New England Journal of } \\
\text { Medicine }\end{array}$ & Letter to the Editor & Princeton, EEUU \\
\hline
\end{tabular}


Frente a la presencia limitada de aportes específicamente dirigidos a la Prostodoncia, se incorporó información que abarcara procedimientos odontológicos trasladables a la Prostodoncia y aquellos que incluyeran procedimientos de control de la infección de superficies inanimadas.

Se agregaron 5 recomendaciones de organismos nacionales e internacionales (MSP, CCl, entre otros) y 5 artículos obtenidos a partir de referencias a publicaciones surgidas en la búsqueda.

Se exponen a continuación sugerencias para el control de la infección, enmarcadas en recomendaciones vigentes y en las emergentes asociadas a la COVID-19.

\section{Recomendaciones generales}

Las recomendaciones actuales destacan la importancia de: a) disponer de información sobre la transmisión del SARS Cov-2 y emplear adecuadamente las barreras de protección o Equipo de Protección Individual (EPI) , b) fomentar el lavado de manos, c) realizar una evaluación detallada del paciente, d) incorporar la aislación con goma dique cuando se maneja turbina, e) emplear sistema anti retracción para instrumental rotatorio, f) solicitar al paciente que efectúe enjuague bucal antes de los procedimientos $y, g$ ) desinfectar todas las superficies dentro de la clínica y espacios asociados $(2,3,9,11,15,16,21,23)$. Estos puntos fueron abordados en los artículos anteriores.

Las sugerencias que se presentan a continuación se destinan tanto al Odontólogo/a Prostodoncista como al resto del equipo de la salud, fundamentalmente al Laboratorista en Odontología y, si bien se concentran en el período de pandemia, también involucran pautas para luego de su finalización. Por ambiente de trabajo se entenderá tanto el espacio clínico como el laboratorio.

\section{La urgencia Odontológica}

Se ha encontrado que durante la pandemia puede variar la demanda de urgencia en cantidad y calidad. La experiencia en otros países muestra que posiblemente ciertas demandas aumenten (lesiones dentino pulpares/periapicales y celulitis 0 abscesos) y otras disminuyan (24). Algunos autores sugieren que la suspensión de la asistencia programada incrementa el volumen de algunas urgencias ${ }^{(25)}$ y otros que luego del cese de las medidas de contingencia, aumentará el número de consultas exponencialmente ${ }^{(24)}$.

Los protocolos indagados consideran como urgencia a las situaciones que involucran dolor dentino pulpar, traumatismo y celulitis o abscesos y excluyen en general a las protésicas ${ }^{(8,22)}$. La ruptura de aparatos se incorpora, en un estudio, como urgencia odontológica asociada a casos oncológicos y a la función 
comprometida (12) y en un protocolo, como eventual tipo de urgencia ${ }^{(3)}$. Otro estudio explícitamente la excluye en el protocolo durante la pandemia ${ }^{(8)}$.

Una sola publicación incorpora recomendaciones que pueden asociarse a procedimientos prostodóncicos, a saber: maximizar esfuerzos para evitar náuseas del paciente (uso de eyectores y cubetas de impresión del tamaño más pequeño posible), uso de goma dique en los procedimientos que involucren instrumental rotatorio (preparación dentaria) y extremar los cuidados de contacto de superficies cuando se realizan retiro y colocación de prótesis removibles ${ }^{(21)}$. Asimismo, un protocolo incluye recomendaciones de manejo de impresiones ${ }^{(3)}$.

\section{Recomendaciones especificas}

En relación al SARS Cov-2, considerando, a) los estudios científicos sobre el período de incubación y las vías de transmisión, b) la experiencia transitada en otros países y, c) los protocolos propuestos por órganos de asesoramiento, se recomienda durante la pandemia:

1. Extremar las medidas de protección, suspender los procedimientos de rutina asistiendo únicamente los casos que constituyan urgencia y minimizar procedimientos invasivos y/o que requieran el uso de spray o aerosoles $(2,8,11)$.

2. Efectuar la higiene del ambiente de trabajo antes y después de cada atención con desinfectantes que contengan entre $62-71 \%$ de Etanol, 0,5\% de Peróxido Hidrogenado y/o 0,1\% de Hipoclorito de Sodio ${ }^{(22-23,26)}$. Todo ambiente de trabajo (sala de espera, consultorio, laboratorio, sala de desinfección) deberá ser considerado potencialmente infectado por lo cual es recomendable preparar para cada jornada laboral una solución desinfectante, con comprobada acción contra el SARS Cov-2, para descontaminar las superficies antes y después de la atención $(10,15)$. Asimismo, el sistema de acondicionamiento del aire se debe desinfectar periódicamente y mantener el entorno limpio y libre de humedad $(11,15)$. Considerando la saliva como vector de transmisión (18,26), todo procedimiento que involucre el retiro y colocación de prótesis en boca y toma de impresiones, requiere un estricto control de la infección en el consultorio y áreas destinadas a trabajos de laboratorio. En tal sentido, antes y después de la asistencia al paciente todas las superficies deberán ser apropiadamente desinfectadas. Las unidades dentales, y sectores directamente asociados, deben desinfectarse antes y después de la atención con soluciones desinfectantes de uso hospitalario como Hipoclorito de Sodio al 0,1\%, Amonios Cuaternarios, Etanol u otros, según la superficie ${ }^{(27)}$. En el área de Laboratorio, utilizar pantallas acrílicas de pulido y sistema de aspiración para controlar la generación de polvo y partículas. 
3. Aplicar protocolo triage, consentimiento informado específico para este escenario y medidas que fomenten la higiene oral. Estos procedimientos siguen las pautas recomendadas para el manejo de urgencias y fueron previamente descriptos en los artículos anteriores. En lo posible, iniciar telefónicamente o por teleconferencia el cuestionario, permitiendo una mejor orientación del estado de salud del paciente ${ }^{(8,9,15,22,28,29)}$ y realizar medición rutinaria de temperatura a pacientes, acompañantes y resto del

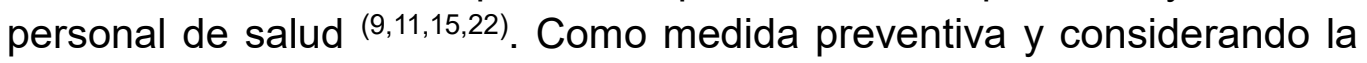
presencia de agentes detergentes en la pasta dental, se recomienda fortalecer los procedimientos de educación para la salud que orienten a los pacientes y a las personas que los cuidan en profundizar el cepillado bucal con pasta dental dos veces al día (30) así como la higiene de las prótesis con agentes que contengan Peróxido de Hidrógeno o Hipoclorito de Sodio. La Historia Clínica deberá incluir un detallado registro del estado de salud general del paciente; se le solicitará el uso de tapabocas cuando sea posible y, de ser necesario toser o estornudar, asegurarse que lo realice sobre un pañuelo descartable ${ }^{(22,26)}$.

4. Sala de espera. Organizar la agenda procurando no concentrar pacientes, concurrencia sin acompañante, eliminación al máximo los artículos de papelería y en el caso que deba asistir un acompañante, se sugiere otorgar un tapabocas si no lo trae consigo $(3,11,26)$. Las superficies de la sala de espera deben limpiarse y luego desinfectarse con los agentes que

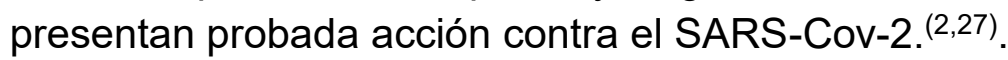

5. Lavado de manos del equipo de salud, del paciente y acompañante, antes y después de la consulta ${ }^{(2,9-11,15,26)}$ extremando cuidados de tocarse la cara y los ojos ${ }^{(15)}$. Disponer de gel hidroalcohólico en sala de espera $(9,10,26)$. Se recomienda, asimismo, la secuencia de lavado de manos y posterior desinfección con gel hidroalcohólico por 20 segundos ${ }^{(3)}$.

6. Utilizar barreras de protección EPI durante los procedimientos clínicos y de laboratorio, siguiendo recomendaciones de secuencia para colocación y retiro $(2,8,15,22)$. Se recomienda: a) para el equipo de salud en el entorno clínico, protección primaria: gorro, lentes protectores, tapaboca o careta, guantes descartables, ropa de trabajo habitual; b) para el odontólogo y personal de apoyo en procedimiento general, protección secundaria: gorro descartable, tapabocas quirúrgico descartable, guantes descartables, lentes protectores, careta, ropa de trabajo y ropa de aislamiento descartable impermeable ${ }^{(3,9)}$. No se aconseja la atención de pacientes con enfermedad constatada en el consultorio particular, sugiriéndose su derivación a servicios de atención debidamente preparados ${ }^{(15)}$. Recomendaciones más específicas de uso de tapabocas se asocian a la asistencia de paciente sintomático (3).

7. Disminución de la carga microbiana. Uso de colutorio bucal previo a iniciar la consulta con Peróxido de Hidrógeno al $1 \%$ (5 mL de Peróxido de 
Hidrógeno $10 \mathrm{Vol}$. adicionando $10 \mathrm{~mL}$ de agua destilada) o Povidona Yodada al $0,2 \%$ (en este último, si se descarta alergia al yodo) ${ }^{(3,8,9,15,22)}$.

8. Toma de impresiones. Se estima que el tiempo de sobrevida del SARS Cov-2 sobre superficies de acero y plástico es de 5.6 hs y 6.8 hs respectivamente (31). Uso de cubetas estériles y desinfección de impresiones. Evitar al máximo los procedimientos que aumenten el riesgo de ahogo o náuseas del paciente. Especial cuidado deberá tenerse en la selección de cubetas y en el manejo adecuado de materiales de impresión (11). Lavado y descontaminación de impresiones según recomendación del fabricante, con agentes que presenten eficacia probada contra el SRAS Cov-2. Si bien se considera que el virus se inactiva luego de 5 minutos de expuesto al Hipoclorito de Sodio de uso doméstico ${ }^{(16)}$, para impresiones de alginato se recomienda desinfección con este agente diluído en agua al $1 \%$ (1:5) por 10 minutos ${ }^{(3)}$.

9. Disponer de recipientes específicos para la colocación de prótesis o impresiones, estériles o desinfectados con agentes que presenten eficacia probada contra el SARS cov-2 (27).

10. Disponer de un espacio para la realización de procedimientos de laboratorio, idealmente separado del ambiente del consultorio y del destinado a desinfectar y esterilizar el instrumental odontológico $(2,17)$. Utilizar pantallas o cámaras de pulido con sistema de aspiración.

11.Prótesis en uso. Antes de proceder a su reparación, lavado del aparato con agua y detergente y posterior desinfección con agentes que presenten eficacia probada contra el SARS Cov-2. Reiterar este procedimiento luego de realizar las etapas de laboratorio (17).

12. Priorizar procedimientos que involucren reparaciones en un acto clínico, efectuando modelos en silicona y no en yeso.

13. Dosificar material de pulido (por ejemplo, pasta de piedra pómez) para cada procedimiento y descartar luego de finalizar (17).

14. Desinfectar fresones, tazas de goma y demás aditamentos una vez culminado el procedimiento de reparación, con agentes que presenten eficacia probada contra el SARS Cov-2 (17). Durante las reparaciones, evitar el uso de instrumental con los que sea más difícil garantizar una total desinfección (por ejemplo, cepillos y pinceles). Utilizar los que se puedan descartar (como lijas) y priorizar el uso los que se pueden esterilizar (fresones).

15. Retiro de las EPI según protocolo ${ }^{(3)}$. Disponer de un adecuado sistema de eliminación de residuos contaminados (15) siguiendo protocolos vigentes ${ }^{(32)}$.

16. Racionalizar el número de visitas. En la medida de lo posible, intentar resolver las reparaciones en la misma sesión y/o emplear procedimientos simplificados que disminuyan el número de visitas al Odontólogo ${ }^{(3)}$. 
Las siguientes recomendaciones fueron realizadas a partir de una revisión no sistematizada por lo que pueden incorporar ciertos sesgos. No obstante ello, se incluyeron estudios provenientes de dos bases de datos junto con protocolos de organismos de asesoramiento nacional e internacionales. A su vez, el documento fue colectivizado con docentes del área, cuyos valiosos aportes fueron incorporados.

Si bien es significativo el volumen de información reunida en los primeros meses de 2020 , es posible sostener que, hasta el momento, es limitada la evidencia científica en el área de la Odontología lo que dificulta generar consensos de aplicación universal $(8,11,15,33)$. En su mayoría, los aportes provienen de estudios secundarios, revisiones de aportes y/o experiencias luego de pasada la pandemia $(3,10,15,16,18,24)$. Son casi inexistentes las contribuciones dirigidas a la Prostodoncia $(3,33)$, lo que generó la necesidad de rescatar procedimientos de protocolos previos a la COVID-19 pero adaptados a la misma ${ }^{(17)}$. A pesar de este escenario de relatividad de la información, es esencial, tanto para el profesional como para la comunidad en general, conocer las vías de transmisión del SARS Cov-2, ya que esto se asocia con una mejor predisposición al seguimiento de los protocolos y al control de la infección ${ }^{(15,34)}$. El carácter impredecible de este proceso incrementa el riesgo de daño ${ }^{(35)}$ por lo que se aconseja enfáticamente seguir las recomendaciones actuales. Éstas, además, requerirán una revisión permanente así como investigaciones focalizadas en el área de la Odontología en general y de la Prostodoncia en particular a fin de nutrir con mayor evidencia al protocolo presentado.

Los rápidos cambios que se suceden durante la contingencia sanitaria determinan la necesidad de una permanente reorganización y adaptación ${ }^{(36)}$. En este sentido, los profesionales podrían introducir la telemedicina para fortalecer la práctica en momentos de pandemia ${ }^{(29)}$ y para implementar procedimientos de información y educación para la salud ${ }^{(37)}$.

Durante esta fase existe consenso en postergar las consultas programadas manteniendo las urgencias, identificadas éstas como las que se presentan con dolor y traumatismo ${ }^{(2,11,24)}$ y excluyendo así a las asociadas con rupturas de prótesis removibles o con la necesidad imperiosa de realizar nuevos aparatos ${ }^{(8)}$. Entendiendo el impacto que la pérdida dentaria tiene en la vida de los sujetos ${ }^{(38-}$ 41), no disponer de orientaciones específicas para el área de la Prostodoncia en procedimientos que no involucren el uso de aerosoles representa una debilidad, sobre todo en contextos de distanciamiento social que no involucran una cuarentena obligatoria. Por ello, el Odontólogo Prostodoncista deberá tener muy claro que, más allá de los aerosoles que puedan o no generarse, la mucosa bucal en general y la saliva en particular, constituyen un vector de transmisión del 
SARS Cov-2 por lo que el conocimiento y aplicación de medidas tendientes a su control son de alto rigor ${ }^{(18-21)}$.

Guo et al encontraron una disminución de urgencias prostodóncicas durante la pandemia, lo que permitiría espaciar consultas y no concentrar personas favoreciendo tiempos de desinfección entre pacientes (24). Sin embargo, es necesario valorar aspectos epidemiológicos en cada país, pudiendo esperarse un comportamiento diferente en Uruguay. Los adultos mayores, quienes presentan alto riesgo de desencadenar peor desenlace por la enfermedad ${ }^{(42,43)}$, podrían requerir asistencia prostodóncica en esta fase. Es esperable, además, que concurran acompañados, aspecto a considerar en la planificación de la consulta, en la organización de la sala de espera y en la implementación de medidas educativas ${ }^{(3,44)}$.

Existe evidencia de que el SARS Cov-2 permanece por horas y hasta días sobre superficies inanimadas ${ }^{(31)}$. Considerando al equipo de salud en el área de la Prostodoncia, debe tenerse especial precaución en el control del ambiente de trabajo, en los procedimientos de recortes y pulidos y en la desinfección de impresiones, prótesis y material asociado (frascos de acrílico, cepillos, fresones, etc.).

Se sugiere extremar las medidas de desinfección de impresiones evitando postergar este procedimiento una vez que ya se obtuvo el modelo de yeso, en el entendido de que los microorganismos pueden penetrar al interior de su estructura manteniéndose viables hasta por una semana (34). Los protocolos vigentes (previos a la pandemia) muestran muchas opciones de tratamiento de las impresiones de alginato, incluyendo el uso de peróxidos, aldehídos, Hipoclorito de Sodio o únicamente lavado con agua y detergente (45-49). Este último no es considerado un método efectivo para desinfección (50). Para el caso de procedimientos que incluyen Hipoclorito de Sodio de uso doméstico, se ha propuesto la dilución en agua 1 en $10^{(51,52)}$ por 5 minutos ${ }^{(51)}$. En el actual escenario, el único protocolo que se detiene en este aspecto, sugiere pulverizar la impresión de alginato con Hipoclorito de Sodio al 1\% (1 en 5) y mantener en bolsa sellada por 10 minutos ${ }^{(3)}$, tiempo considerado por algunos ideal ${ }^{(34)}$. En relación al método de desinfección, la pulverización otorga la posibilidad de menores modificaciones dimensionales en el alginato y permite un menor gasto del agente, mientras que, la inmersión garantiza el acceso del desinfectante a todas las superficies de la impresión pero ofrece mayor riesgo de cambios dimensionales ${ }^{(53,54)}$, factor a considerar si se duplica el tiempo de desinfección. Ulgey, Gorley y Yesilyurt sostienen que existe suficiente evidencia como para preferir la inmersión sobre la pulverización y agregan que debe realizarse lo más pronto posible luego de efectuada la técnica ${ }^{(54)}$. Teniendo en cuenta la escasa información presente basada en evidencia científica, se recomienda actuar con celeridad en el lavado de la impresión con agua y 
detergente, enjuagarla y proceder a su desinfección sumergiéndola en una solución de Hipoclorito de Sodio al $1 \%$ por 10 minutos.

No se encontró información sobre descontaminación de aparatos dirigida al control de la transmisión SARS Cov-2. Según lo aportado por Kampf et al, es posible inactivar el virus presente en superficies con Etanol 62-71\%, 0,5\% de Hipoclorito de Sodio al $0,1 \%$ por 1 minuto ${ }^{(14)}$. No obstante, su estudio no involucra acrílicos protésicos que presenten cierto grado de porosidad y consecuente penetración del virus. El Hipoclorito de Sodio es un agente de uso frecuente en desinfección de prótesis removible (55); es necesario realizar estudios que permitan revisar los protocolos vigentes a fin de valorar su efectividad contra el SARS Cov-2. Para las prótesis removibles, se sugiere el procedimiento de lavado con agua y detergente y desinfectar con agentes de comprobada acción contra SARS Cov-2.

Si bien existen autores que sostienen que la dinámica de las urgencias podría mostrar variaciones en esta fase y que la demanda de atención aumentaría exponencialmente luego de la pandemia ${ }^{(24)}$, las condiciones socio-económicas de la población podrían marcar un viraje hacia servicios colectivizados. La información recabada en relación a protocolos en servicios colectivizados (con y sin función de enseñanza) proviene de Hospitales Odontológicos o Médicos con funcionamiento Odontológico (24). Por tal motivo, las recomendaciones no pueden trasladarse directamente a situaciones como las de la Facultad de Odontología Udelar. Otras variables deberán ser consideradas como: el acondicionamiento de los espacios para la descontaminación del instrumental, el rol que desempeña el personal de apoyo, la adecuación de horarios para desinfección de espacios clínicos, uso racional de espacios físicos limitando concentración de personas, la implementación de tele-consultas y la coordinación y dosificación del número de estudiantes en el área clínica, entre otros.

\section{Síntesis final}

Este documento presentó una recomendación de actuación frente a la urgencia prostodóncica aplicable a la práctica odontológica general y especializada. Aspectos más abarcativos del control de la infección y procedimientos desarrollados en otras áreas de la Odontología fueron profundamente analizados en los artículos anteriores.

El dinamismo y la incertidumbre continúan siendo la esencia de esta fase de pandemia. Se destaca así la necesidad de avanzar en el acuerdo de procedimientos seguros, específicos, con base en la evidencia científica y en procura de proyectar procedimientos para "el día después" contemplando el ejercicio liberal y colectivizado de la profesión así como la asistencia en contexto de enseñanza. 
Agradecimientos

A los colegas docentes: Raúl Riva, Juan Pablo Poeymiró, Federico Riva, José Regueira, Rodrigo Fuentes, Germán Puig y Gabriel Castellanos cuyos valiosos aportes enriquecieron este artículo.

Referencias

1. Uruguay. Ministerio de Salud Pública. Sistema Nacional Integrado de Salud. Anexo II. Catálogo de prestaciones. Montevideo: Ministerio de Salud Pública - Uruguay. 2008.

2. Estados Unidos de Norteamérica. CDC. Coronavirus Disease 2019 ( COVID-19 ) Infection Control Guidance for Healthcare Professionals about Coronavirus ( COVID-19 ). 2020.

3. Organización Colegial de Dentistas de España. Consejo Dentistas. PLAN ESTRATÉGICO DE ACCIÓN PARA EL PERIODO POSTERIOR A LA CRISIS CREADA. Madrid. 2020.

4. Estados Unidos de Norteamérica. CDC. Interim Infection Prevention and Control Recommendations for Patients with Suspected or Confirmed Coronavirus Disease 2019 (COVID-19) in Healthcare Settings [Internet]. Vol. 2, CDC. 2020: 1-10. Disponible en: https://www.cdc.gov/coronavirus/2019-ncov/infection-control/controlrecommendations.html

5. Sohrabi C, Alsafi Z, Neill NO, Khan M, Kerwan A. World Health Organization declares global emergency: A review of the 2019 novel coronavirus (COVID-19). Int J Surg. 2020;76(January):71-6.

6. Rothe C, Schunk M, Sothmann P, Bretzel G, Froeschl G, Wallrauch C, et al. Transmission of 2019-NCOV infection from an asymptomatic contact in Germany. N Engl J Med. 2020;382(10):970-1.

7. Khader Y, Al Nsour M, Al-Batayneh OB, Saadeh R, Bashier $\mathrm{H}$, Alfaqih $M$, et al. Dentists' awareness, perception, and attitude regarding COVID-19 and infection control: A cross-sectional study among Jordanian dentists (Preprint). JMIR Public Heal Surveill Prepr. 2020;(March).

8. Alharbi A, Alharbi S, Alqaidi S. Guidelines for dental care provision during the COVID-19 pandemic. Saudi Dent J [Internet]. 2020; Disponible en: http://www.sciencedirect.com/science/article/pii/S1013905220303266

9. Araya SC. Consideraciones para la Atención de Urgencia Odontológica y Medidas Preventivas para COVID-19 (SARS-CoV 2) Considerations for Emergency Dental Care and Preventive Measures for COVID-19 (SARSCoV 2). Int J Odontostomat [Internet]. 2020;14(3):268-70. Disponible en: https://journals.sagepub.com/doi/full/10.1177/

10.Spagnuolo G, De Vito D, Rengo S, Tatullo M. COVID-19 outbreak: An overview on dentistry. Int J Environ Res Public Health. 2020;17(6):1-4. 
11. Meng L, Hua F, Bian Z. Coronavirus Disease 2019 (COVID-19): Emerging and Future Challenges for Dental and Oral Medicine. J Dent Res. 2020.

12. Sepúlveda Verdugo C, Secchi Álvarez A, Donoso-Hofer F. Urgency Dental Care Considerations in the Context of Coronavirus COVID-19 (SARSCoV-2). Int J Odontostomat [Internet]. 2020;14(3):279-84. Disponible en: http://www.ijodontostomatology.com/wpcontent/uploads/2020/03/2020 v14n3 003.pdf

13. Belser JA, Rota PA, Tumpey TM. Ocular Tropism of Respiratory Viruses. Microbiol Mol Biol Rev. 2013;77(1):144-56.

14. Kampf G, Todt D, Pfaender S, Steinmann E. Persistence of coronaviruses on inanimate surfaces and their inactivation with biocidal agents. J Hosp Infect [Internet]. 2020;104(3):246-51. Disponible en: https://doi.org/10.1016/j.jhin.2020.01.022

15. Peng X, Xu X, Li Y, Cheng L, Zhou X, Ren B. Transmission routes of 2019$\mathrm{nCoV}$ and controls in dental practice. Int $\mathrm{J}$ Oral Sci [Internet]. 2020;12(1):1-6. Disponible en: http://dx.doi.org/10.1038/s41368-0200075-9

16. Aquino-canchari CR. COVID-19 y su repercusión en la Odontología COVID-19 and its impact on dental care. 2020;57(1):2-4.

17. Emmerson AM. ADA's Guidelines for infection control. 3rd ed. Research and Clinical Forums. Australian Dental Association; 2015.

18. Sabino-Silva R, Jardim ACG, Siqueira WL. Coronavirus COVID-19 impacts to dentistry and potential salivary diagnosis. Clin Oral Investig. 2020;13-5.

19. Khurshid Z, Asiri FYI, AI Wadaani H. Human Saliva: Non-Invasive Fluid for Detecting Novel Coronavirus (2019-nCoV). Int J Environ Res Public Health. 2020;17(7):17-20.

20. Xu H, Zhong L, Deng J, Peng J, Dan H, Zeng X, et al. High expression of ACE2 receptor of 2019-nCoV on the epithelial cells of oral mucosa. Int $J$ Oral Sci [Internet]. 2020;12(1):1-5. Disponible en: http://dx.doi.org/10.1038/s41368-020-0074-x

21. Ge Z, Yang L, Xia J, Fu X, Zhang Y. Possible aerosol transmission of COVID-19 and special precautions in dentistry. J Zhejiang Univ B [Internet]. 2020. Disponible en: https://doi.org/10.1631/jzus.B2010010

22. Ather A, Biraj Patel, Ruparel NB, Diogenes A, Hargreaves KM. Coronavirus Disease 19 (COVID-19): Implications for Clinical Dental Care. J Endod [Internet]. 2020;46(5):2020. Disponible en: https://doi.org/10.1016/j.joen.2020.03.008

23. MSP. Protocolo de condiciones y medio ambiente de trabajo. Medidas de protección del equipo de salud y usuarios del SNIS. MSP. 2020.

24. Guo H, Zhou Y, Liu X, Tan J. The impact of the COVID-19 epidemic on the utilization of emergency dental services. J Dent Sci [Internet]. 2020:0-3. Disponible en: https://doi.org/10.1016/j.jds.2020.02.002 
25. Dave M, Seoudi N, Coulthard P. Urgent dental care for patients during the COVID-19 pandemic. Lancet [Internet]. 2020;6736(20):30806. Disponible en: $\mathrm{http}: / / d x . d o i . o r g / 10.1016 / S 0140-6736(20) 30806-0$

26. Prati C, Pelliccioni GA, Sambri V, Chersoni S, Gandolfi MG. COVID-19: its impact on dental schools in Italy, clinical problems in endodontic therapy and general considerations. [Internet]. Vol. 53, International endodontic journal. 2020. 723-5. Disponible en: http://www.ncbi.nlm.nih.gov/pubmed/32277770

27. EPA United States Enviromental Protection Agency. List N: Disinfectants for Use Against SARS-CoV-2. EPA.gov. 2020.

28. Yang Y, Zhou Y, Liu X, Tan J. Health services provision of 48 public tertiary dental hospitals during the COVID-19 epidemic in China. Clin Oral Investig. 2020.

29. Machado RA, de Souza NL, Oliveira RM, Martelli Júnior $H$, Bonan PRF. Social media and telemedicine for oral diagnosis and counselling in the COVID-19 era. [Internet]. Oral oncology. Elsevier. 2020. Disponible en: http://www.ncbi.nlm.nih.gov/pubmed/32291154

30. Bristol MA. Toothbrushing against coronavirus. Vol. 228, British Dental Journal. 2020. p. 487.

31. van Doremalen N, Bushmaker T, Morris DH, Holbrook MG, Gamble A, Williamson BN, et al. Aerosol and Surface Stability of SARS-CoV-2 as Compared with SARS-CoV-1. N Engl J Med [Internet]. el 17 de marzo de 2020;382(16):1564-7. Disponible

en: https://doi.org/10.1056/NEJMc2004973

32. Muñiz S, Ardus M, Rodríguez J, Cabrera P, Pereyra N, Millán R. ASSE. Manual de "Gestión Integral de Residuos Sanitarios". 2016.

33. Martelli-Júnior H, Machado RA, Martelli DRB, Coletta RD. Dental journals and coronavirus disease (COVID-19): A current view [Internet]. Oral Oncology. Elsevier. 2020. 104664. Disponible en: https://doi.org/10.1016/j.oraloncology.2020.104664

34. Gaffar BO, El Tantawi M, Al-Ansari AA, Al-AnsariAIAgl AS, Farooqi FA, Almas KM. Knowledge and practices of dentists regarding MERS-CoV: A cross-sectional survey in Saudi Arabia. Saudi Med J. 2019;40(7):714-20.

35. Wynants L, Van Calster B, Bonten MMJ, Collins GS, Debray TPA, De Vos $M$, et al. Prediction models for diagnosis and prognosis of covid-19 infection: systematic review and critical appraisal. BMJ [Internet]. 2020; 369:m1328.

Disponible

en:

http://www.ncbi.nlm.nih.gov/pubmed/32265220

36. Boldrini P, Bernetti A, Fiore P, SIMFER Executive Committee and SIMFER Committee for international affairs. Impact of COVID-19 outbreak on rehabilitation services and Physical and Rehabilitation Medicine (PRM) physicians' activities in Italy. An official document of the Italian PRM Society (SIMFER). Eur J Phys Rehabil Med [Internet]. 2020; Disponible en: http://www.ncbi.nlm.nih.gov/pubmed/32175719 
37. Ambigapathy S, Rajahram GS, Shamsudin UK, Khoo EM, Cheah WK, Peariasamy $\mathrm{KM}$, et al. How should front-line general practitioners use personal protective equipment (PPE)? Malaysian Fam Physician. 2020;15(1):2-5.

38. Peršić S, Čelebić A. Influence of different prosthodontic rehabilitation options on oral health-related quality of life, orofacial esthetics and chewing function based on patient-reported outcomes. Qual Life Res. 2015;24(4):919-26.

39. Montero J, Castillo-Oyague R, Lynch CD, Albaladejo A, Castano A. Selfperceived changes in oral health-related quality of life after receiving different types of conventional prosthetic treatments: a cohort follow-up study. J Dent. junio de 2013;41(6):493-503.

40. Inukai M, John MT, Igarashi Y, Baba K. Association between perceived chewing ability and oral health-related quality of life in partially dentate patients. Health Qual Life Outcomes. octubre de 2010;8:1-6.

41. Visscher CM, Lobbezoo F, Schuller AA. Dental status and oral healthrelated quality of life . A population-based study. J Oral Rehabil. junio de 2014;41(6):416-23.

42. Huenchuan S. COVID-19. Recomendaciones generales para la atención a personas mayores desde una perspectiva de derechos humanos. Ciudad de México: Naciones Unidas CEPAL. 2020.

43. Nicola M, O'Neill N, Sohrabi C, Khan M, Agha M, Agha R. Evidence Based Management Guideline for the COVID-19 Pandemic - Review article. Int J Surg [Internet]. 2020; Disponible en: http://www.ncbi.nlm.nih.gov/pubmed/32289472

44. Montero-Odasso M, Goens SD, Kamkar N, Lam R, Madden K, Molnar F, et al. Canadian Geriatrics Society COVID-19 Recommendations for Older Adults . What Do Older Adults Need To Know ? 2020;23(1):149-51.

45. Carr AB, Brown DT. McCracken's Removable Partial Prosthodontics. 12th ed. St. Louis, Missouri: Elsevier. Mosby. 2011. 224-225.

46. Phoenix RD, Cagna DR, DeFreest CF. Stewart's Clinical. Removable Partial Prosthodontics. 4th. Chicago: Quintessence Publishing Co. Inc.. 2008. 144-145.

47. Todescan R, Bernades da Silva EE, da Silva OJ. Atlas de Prótese Parcial Removível. 5ta. San Pablo: Livraria Santos Editora. 2009. 246-247.

48. Bassi F, Previgliano V, Schierano G. Rehabilitación protésica - Protesi Parziale Rimovibile. 2011a ed. Buenos Aires: AMOLCA. 2011. 94.

49. Mallat Desplats E, Mallat Callís E. Prótesis parcial removible y sobredentaduras. Madrid: Elsevier España, S.A. 2004. 186-187.

50. Junevicius J, Pavilonis A, Surna A. Transmission of Microorganisms from Dentists to Dental Laboratory Technicians through Contaminated Dental Impressions. Balt Dent Maxillofac J. 2004;6(1):20-3.

51. Bocage M. Prótesis Parcial Removible. Montevideo: Barreiro, 2009. 
52. Hemalatha R, Ganapathy D. Disinfection of dental impression- A current overview. J Pharm Sci Res. 2016;8(7):661-4.

53. Al Mortadi N, Al-Khatib A, Alzoubi KH, Khabour OF. Disinfection of dental impressions: Knowledge and practice among dental technicians. Clin Cosmet Investig Dent. 2019;11:103-8.

54. Ulgey M, Gorler O, Yesilyurt G. Importance of disinfection time and procedure with different alginate impression products to reduce dimensional instability. Niger J Clin Pract. 2020;23(3):284-90.

55. De Sousa Porta SR, De Lucena-Ferreira SC, Da Silva WJ, Del Bel Cury AA. Evaluation of sodium hypochlorite as a denture cleanser: A clinical study. Gerodontology. 2015;32(4):260-6. 